\title{
Abundances of planetary nebulae NGC 7662 and NGC 6741^
}

\author{
S. R. Pottasch ${ }^{1}$, D. A. Beintema ${ }^{1,2}$, J. Bernard Salas ${ }^{1,2}$, and W. A. Feibelman ${ }^{3}$ \\ 1 Kapteyn Astronomical Institute, PO Box 800, 9700 AV Groningen, The Netherlands \\ 2 SRON Laboratory for Space Research, PO Box 800, 9700 AV Groningen, The Netherlands \\ 3 Laboratory for Astronomy and Solar Physics, Code 681, Goddard Space Flight Center, MD, USA
}

Received 23 August 2001 /Accepted 19 October 2001

\begin{abstract}
The ISO and IUE spectra of the elliptical nebulae NGC 7662 and NGC 6741 are presented. These spectra are combined with the spectra in the visual wavelength region to obtain a complete, extinction corrected, spectrum. The chemical composition of the nebulae is then calculated and compared to previous determinations. The abundances found are compared to determinations made in other nebulae using ISO data. A discussion is given to see if possible evolutionary effects can be found from the abundance differences.
\end{abstract}

Key words. ISM: abundances - planetary nebulae: individual: NGC 7662, NGC 6741 infrared: ISM: lines and bands

\section{Introduction}

Including the ISO SWS spectra of planetary nebulae with spectra of the nebula in other spectral regions allows an abundance determination which has several important advantages. These have been discussed in earlier papers (e.g. see Pottasch \& Beintema (1999), Pottasch et al. (2000), or Bernard Salas et al. (2001). The most important advantage is that the infrared lines originate from very low energy levels and thus give an abundance which is not sensitive to the temperature in the nebula, nor to possible temperature fluctuations. Furthermore, when a line originating from a high-lying energy level in the same ion is observed, it is possible to determine an effective temperature at which the lines in that particular ion are formed. When the effective temperature for many ions can be determined, it is possible to make a plot of effective temperature against ionization potential, which can be used to determine the effective temperature for ions for which only lines originating from a high energy level are observed. Use of an effective electron temperature takes into account the fact that ions are formed in different regions of the nebula. At the same time possible temperature fluctuations are taken into account.

Use of the ISO spectra have further advantages. One of them is that the number of observed ions used in the

\footnotetext{
Send offprint requests to: S. R. Pottasch, e-mail: pottasch@astro.rug.nl

* Based on observations with ISO, an ESA project with instruments funded by ESA Member States (especially the PI countries: France, Germany, The Netherlands and the UK) and with the participation of ISAS and NASA.
}

abundance analysis is approximately doubled, which removes the need for using large "Ionization Correction Factors", thus substantially lowering the uncertainty in the abundance. A further advantage is that the extinction in the infrared is almost negligible, eliminating the need to include large correction factors.

In much of the previously published analysis of $\mathrm{PN}$ using ISO spectra the nebulae involved had a bipolar morphology. In this paper we discuss two nebulae classified as "elliptical": NGC 7662 and NGC 6741. Their abundances will be compared to the other nebulae in the final section.

\section{The spectrum of NGC 7662}

\subsection{ISO observations}

The ISO SWS observations were made with the SWS02 observing template which gives good spectral resolution for a limited number of lines. This was supplemented by an SWS01 observation. The intensity of the lines found in the spectrum is shown in Table 1. The uncertainty of the stronger lines is about $10 \%$. The measurements were centered at $\mathrm{RA}(2000) 23^{\mathrm{h}} 25^{\mathrm{m}} 53.9^{\mathrm{s}}$ and $\operatorname{Dec}(2000)+42^{\circ} 32^{\prime} 5.4^{\prime \prime}$. The diaphragm used was $15^{\prime \prime} \times$ $20^{\prime \prime}$ below $12 \mu \mathrm{m}$ and somewhat larger above this wavelength. The nebula is somewhat larger than these dimensions. The total radio emission from the nebula is $631 \mathrm{mJy}$ (the $6 \mathrm{~cm}$ flux density given by Zijlstra et al. 1989). This leads to an expected $\mathrm{H} \beta$ intensity of $1.53 \times$ $10^{-10} \mathrm{erg} \mathrm{cm}^{-2} \mathrm{~s}^{-1}$, which in turn leads to an expected $\operatorname{Br} \alpha$ of $1.04 \times 10^{-11} \mathrm{erg} \mathrm{cm}^{-2} \mathrm{~s}^{-1}$ (at $\left.T_{\mathrm{e}}=12000 \mathrm{~K}\right)$. If this is true, the ISO intensity in Table 1 shows that $58 \%$ of 
Table 1. ISO observations of NGC7662 (in units of $10^{-12} \mathrm{erg} \mathrm{cm}^{-2} \mathrm{~s}^{-1}$ ).

\begin{tabular}{|c|c|c|c|c|c|}
\hline Ident. & $\lambda(\mu \mathrm{m})$ & Intens. $^{\dagger}$ & Ident. & $\lambda(\mu \mathrm{m})$ & Intens. $^{\dagger}$ \\
\hline$?$ & 2.555 & 2.8 & [S IV $]$ & 10.51 & 130.8 \\
\hline Н г $6-4$ & 2.626 & 3.7 & {$[\mathrm{Ne}$ II $]$} & 12.81 & 0.78 \\
\hline He II $7-6$ & 3.092 & 2.1 & {$[\mathrm{Mg} \mathrm{V}]$} & 13.52 & $0.5(:)$ \\
\hline$?$ & 3.174 & 4.7 & {$[\mathrm{Ne} \mathrm{V}]$} & 14.32 & 22.6 \\
\hline Н г $8-5$ & 3.742 & 0.8 & [Ne III] & 15.55 & 82.3 \\
\hline Н г $5-4$ & 4.052 & 6.2 & [S III] & 18.72 & 12.4 \\
\hline [Mg IV] & 4.486 & 16.9 & {$[\mathrm{Ne} \mathrm{V}]$} & 24.32 & 24.8 \\
\hline$[\mathrm{Ar} \mathrm{VI}]$ & 4.529 & 1.3 & [O IV] & 25.89 & 463.9 \\
\hline$[\mathrm{Mg} \mathrm{V}]$ & 5.609 & 2.3 & [S III] & 33.47 & 5.0 \\
\hline$?$ & 6.247 & 3.2 & [Ne III] & 36.01 & 9.17 \\
\hline$[\mathrm{Ar} \mathrm{V}]$ & 7.905 & 1.9 & {$[\mathrm{~N} \mathrm{III}]^{*}$} & 57 & 18.6 \\
\hline \multirow[t]{2}{*}{ [Ar III] } & 8.992 & 5.6 & {$[\mathrm{O} \text { II }]^{*}$} & 52 & 205 \\
\hline & & & {$[\mathrm{O} \text { III }]^{*}$} & 88 & 78 \\
\hline
\end{tabular}

$\dagger$ These are aperture corrected intensities (see text).

* These data are from Liu et al. (2001) and refer to the entire nebula.

the nebula is being seen in the $15^{\prime \prime} \times 20^{\prime \prime}$ diaphragm. This is in good agreement with the calculation of Harrington et al. (1982) that $52 \%$ of the $\mathrm{H} \beta$ flux is being seen in the IUE diaphragm which is slightly smaller $\left(10^{\prime \prime} \times 23^{\prime \prime}\right)$. Thus a correction factor of 1.72 was used to obtain the total intensity for the ISO lines below $12 \mu \mathrm{m}$. A slightly lower factor, 1.5, was used for the ISO lines between $12 \mu \mathrm{m}$ and $27 \mu \mathrm{m}$ because of the slightly larger diaphragm used. Above $30 \mu \mathrm{m}$, including the LWS measurements, no correction factor was necessary since the entire nebula was being seen. The LWS measurements are reported and discussed by Liu et al. (2001).

\subsection{Comparison with other infrared observations}

A comparison is made in Table 2. Only four lines have been measured earlier and the comparison is made difficult because different diaphragms were used. The IRAS measurements refer to the entire nebula, and this is probably the case for the measurements of Shure et al. (1983). Increasing the ISO measurements by the factor 1.5 gives good agreement on average for the 3 lines above $12 \mu \mathrm{m}$ and 1.72 appears consistent for the [S IV] line. Because now the [O IV] line intensity is $35 \%$ higher than found by Shure et al., one can consider the possibility that this oxygen line is formed in a region closer to the star and a smaller correction factor is needed. But this is limited by the [Nev] line which would also be formed closer to the star and requires that the correction factor not be too small to get agreement with the measurements of Shure et al. A correction factor of 1.4 for the high ionization stages, and 1.6 for the low ionization stages might give better agreement. But because the differences are less than $10 \%$ we have chosen not to use this more complicated variant.
Table 2. NGC 7662: comparison of infrared observations (in units of $\left.10^{-12} \mathrm{erg} \mathrm{cm}^{-2} \mathrm{~s}^{-1}\right)$.

\begin{tabular}{clrlllll}
\hline$\lambda$ & Ident. & ISO & \multicolumn{2}{c}{ IRAS } & GMS & B & S \\
\cline { 3 - 5 }$(\mu \mathrm{m})$ & & & $(1)$ & $(2)$ & $(3)$ & $(4)$ & $(5)$ \\
\hline 15.55 & {$[\mathrm{Ne} \mathrm{III}]$} & 82.3 & & 147 & & & \\
10.51 & {$[\mathrm{~S} \mathrm{IV}]$} & 130.8 & 100 & 188 & 90 & 67 & \\
24.32 & {$[\mathrm{Ne}]$} & 24.8 & & & & & 35.8 \\
25.89 & {$[\mathrm{O}$ IV $]$} & 463.9 & & & & & 514 \\
\hline
\end{tabular}

(1) Pottasch et al. (1986); (2) re-reduced measurements; (3) Gillett et al. 11" diaphragm; (4) Beck et al. (1981) 6" diaphragm: (5) Shure et al. (1983) 30" diaphragm.

\subsection{Extinction}

The observed $6 \mathrm{~cm}$ radio emission (631 mJy, Zijlstra et al. 1989) predicts a total $\mathrm{H} \beta$ flux for the nebula of $1.53 \times 10^{-10} \mathrm{erg} \mathrm{cm}^{-2} \mathrm{~s}^{-1}$. The observed $\mathrm{H} \beta$ is $1.02 \times 10^{-10}$ (Cahn et al. 1992). The extinction is therefore $c=0.176$. The observed Balmer decrement is consistent with this value: Cahn et al. (1992) give $c=0.15$ and Stasinska et al. (1992) find $c=0.20$. We will use $c=0.18$ or $E_{B-V}=0.123$.

\subsection{Comparison of ISO hydrogen and ionized helium lines with theory}

A comparison of the ISO hydrogen and ionized helium lines with theory is made in Table 3 . This is useful especially to determine the amount of these ions which are seen through the ISO aperture. As can be seen from the table, three infrared hydrogen lines can be seen in the ISO spectrum. There intensities are given in the third column of the table, and corrected for a very small extinction in the fourth column. The theoretical values are shown in the fifth column and are taken from Hummer and Storey (1987) for $T_{\mathrm{e}}=12500 \mathrm{~K}$ and $n_{\mathrm{e}}=10^{4} \mathrm{~cm}^{-3}$. The values are adjusted to give the best fit. At the same time this fixes the intensity of all other hydrogen lines, in particular the $\mathrm{H} \beta$ line. The intensity found for this line is about $59 \%$ of the total $\mathrm{H} \beta$, indicating that the ISO diaphragm is seeing $59 \%$ of the nebular flux. It may be noticed that the relative intensities of the observed infrared lines is in good agreement with the theoretical expectation. The same can be done for the ionized helium line. Since only a single line is observed, its intensity fixes the intensities of the helium lines in the visible and ultraviolet part of the spectrum $\lambda 4686 \AA$ and $\lambda 1640 \AA$. The latter is of importance in comparing the IUE spectrum with ISO and visible spectrum. It should be noted that the ratio of $\lambda 4686 \AA$ to $\mathrm{H} \beta$ found in this way, 0.67 , is in very good agreement with the ratio observed in the visible spectrum by Hyung \& Aller (1997a). 
Table 3. NGC 7662: hydrogen and ionized helium line intensities (in units of $10^{-12} \mathrm{erg} \mathrm{cm}^{-2} \mathrm{~s}^{-1}$ ).

\begin{tabular}{lcccc}
\hline$\lambda(\mu \mathrm{m})$ & Transit. & Obs. & Corr. & Theory \\
\hline 4.052 & $\operatorname{Br} \alpha(5-4)$ & 6.2 & 6.25 & 6.31 \\
2.626 & $\operatorname{Br} \beta(6-4)$ & 3.7 & 3.77 & 3.68 \\
3.742 & $\operatorname{Pf} \gamma(8-5)$ & 0.8 & 0.82 & 0.86 \\
$4861 \AA$ & $\operatorname{H} \beta$ & & & 89.8 \\
& & & & \\
3.092 & $\operatorname{He} I I(7-6)$ & 2.1 & 2.12 & 2.12 \\
$4686 \AA$ & $\operatorname{He} I I(4-3)$ & & & 60.4 \\
$1640 \AA$ & $\operatorname{HeII}(3-2)$ & & & 425 \\
\hline
\end{tabular}

The theoretical values assume an electron temperature for hydrogen of $T_{\mathrm{e}}=12500 \mathrm{~K}$, and for ionized helium of $T_{\mathrm{e}}=15000 \mathrm{~K}$.

\subsection{The visual and the ultraviolet spectrum}

The visual spectrum used is that of Hyung \& Aller (1997a). This spectrum refers to a small $\left(6^{\prime \prime} \times 4^{\prime \prime}\right)$ region on the bright ring of the nebula. We assume that the spectrum is similar over the entire nebula. This is an approximation which is reasonable but not perfect. It would have been preferable to have the spectrum measured through a diaphragm as large as the SWS diaphragm. Thus for the visual spectrum we use the intensities measured by Hyung and Aller, corrected for an extinction of $c=0.18$, and normalized so that $\mathrm{H} \beta=1.53 \times 10^{-10} \mathrm{erg} \mathrm{cm}^{-2} \mathrm{~s}^{-1}$.

A measurement of the flux of the $[\mathrm{NeV}]$ line at $\lambda 3426 \AA$ has been made by Rowlands et al. (1984) using a $48^{\prime \prime}$ diaphragm which includes the entire nebula. They find a flux of $5.9 \times 10^{-12} \mathrm{erg} \mathrm{cm}^{-2} \mathrm{~s}^{-1}$ for this line.

The IUE spectrum is shown in Table 4 . The first and second column of this table give the wavelength and identification of the observed lines. The third column gives the intensity measured from the high resolution spectra SWP 04106 and LWR 06805. The fourth column is the intensity measured from the low resolution spectra SWP 06408, SWP 03812 and LWR 02657. All these spectra are taken with the central star in the diaphragm. We have also looked at low resolution spectra taken $7^{\prime \prime}$ away from the central star (SWP 06466 and LWR 05548) and find good agreement with the measurements presented.

The fifth column of the table gives the intensity through the IUE diaphragm after correction for extinction. Finally, the last column gives the total intensity of the nebula, normalized so that the He II $\lambda 1640 \AA$ has the intensity predicted by theory and the measurement of $\lambda 4686 \AA$ in the visible and the $3.092 \mu \mathrm{m}$ line in the infrared. Thus the intensities in all three wavelength regions refer to the entire nebula and are compatible with each other, with the understanding that the intensities in Table 1 (below $27 \mu \mathrm{m}$ ) and Table 3 refer to $58 \%$ of the total intensity.
Table 4. IUE Spectrum of NGC 7662 .

\begin{tabular}{|c|c|c|c|c|c|}
\hline \multirow{2}{*}{$\begin{array}{l}\lambda \\
(\AA)\end{array}$} & \multirow[t]{2}{*}{ Ion } & \multicolumn{3}{|c|}{ Intensities } & \multirow{2}{*}{$\begin{array}{l}\text { Norm. } \\
\text { Intens. }\end{array}$} \\
\hline & & (1) & $(2)$ & (3) & \\
\hline 1175.60 & $\mathrm{C}_{\mathrm{III}}{ }^{\natural, *}$ & 2.41 & & 7.95 & 23.3 \\
\hline 1238.69 & $\mathrm{Nv}$ & 0.78 & & & \\
\hline 1242.66 & $\mathrm{NV}$ & 0.17 & 2.2 & 6.64 & 19.5 \\
\hline 1372.15 & $\mathrm{Ov}$ & 1.62 & & & \\
\hline 1393.65 & Si IV & 0.82 & & & \\
\hline 1399.58 & O IV] & 0.93 & 6.9 & 15.8 & 46.4 \\
\hline 1400.98 & O IV] & 3.06 & & & \\
\hline 1404.61 & O IV] & 2.19 & & & \\
\hline 1407.23 & O IV] & 0.74 & & & \\
\hline 1483.13 & $\mathrm{~N}$ IV] & 3.70 & 5.3 & 13.7 & 40.1 \\
\hline 1486.53 & N IV] & 1.93 & & & \\
\hline 1549 & $\mathrm{CIV}^{\natural}$ & & 168. & 423. & 1240. \\
\hline 1640 & He II ${ }^{\natural}$ & & 98. & 243. & 713. \\
\hline 1660.62 & O III] & 1.50 & & & \\
\hline 1666.01 & O III] & 4.70 & 5.1 & 13.1 & 38.4 \\
\hline 1746.78 & N III] & 0.08: & & & \\
\hline 1748 & N III] & $0.13:$ & & & \\
\hline 1749.49 & N III] & 1.15 & 1.86 & 4.62 & 13.5 \\
\hline 1752.00 & N III] & 0.76 & & & \\
\hline 1753.69 & N III] & $0.11:$ & & & \\
\hline 1882.41 & Si III] & 1.21 & 1.2 & 2.9 & 8.5 \\
\hline 1909 & C III] & & 94. & 235. & 689. \\
\hline 2253 & HeII ${ }^{\natural}$ & 0.42 & & 1.2 & 3.5 \\
\hline 2297 & $\mathrm{C}_{\mathrm{III}}^{\mathrm{\natural}, *}$ & 2.47 & 3.25 & 7.1 & 20.8 \\
\hline 2306.9 & HeII ${ }^{\natural}$ & 0.71 & & 2.0 & 6.9 \\
\hline $2325^{\dagger}$ & [O III], C II] & & 3.65 & 9.75 & 28.6 \\
\hline 2385 & He II & 1.3 & 1.14 & 3.1 & 9.1 \\
\hline 2422 & {$[\mathrm{Ne} I \mathrm{IV}]$} & 12.0 & & 29.2 & 85.8 \\
\hline 2424 & [Ne IV] & 13.6 & & 33.0 & 96.7 \\
\hline 2468.7 & {$[\mathrm{O} \quad \mathrm{II}]$} & 0.27 & & 0.63 & 1.85 \\
\hline 2511 & He II ${ }^{\natural}$ & 1.95 & 2.97 & 6.4 & 18.8 \\
\hline 2733 & He II ${ }^{\natural}$ & & 5.63 & 11.2 & 32.8 \\
\hline 2783.6 & {$[\mathrm{Mg} \mathrm{v}]$} & 0.37 & & 0.74 & 2.2 \\
\hline 2854 & [Ar IV] & 0.73 & & 1.5 & 4.4 \\
\hline 2869 & [Ar IV] & 0.28 & & 0.55 & 1.6 \\
\hline 3024 & O III & 1.57 & 1.75 & 3.2 & 9.4 \\
\hline 3047 & O III & 5.06 & 6.92 & 12.7 & 37.2 \\
\hline 3133 & O III & 30.0 & & 56. & 164 \\
\hline 3203 & He II & 7.93 & 10.9 & 19.1 & 56. \\
\hline
\end{tabular}

(1) From SWP 04106 and LWR 06805 - all intensity units $10^{-12} \mathrm{erg} \mathrm{cm}^{-2} \mathrm{~s}^{-1}$.

(2) From low resolution intensity SWP 06408 SWP 0312 and LWR 02657 - all intensity units $10^{-12} \mathrm{erg} \mathrm{cm}^{-2} \mathrm{~s}^{-1}$.

(3) Low resolution intiensities corrected for extinction, $E_{B-V}=0.123$ - all intensity units $10^{-12} \mathrm{erg} \mathrm{cm}^{-2} \mathrm{~s}^{-1}$.

$\dagger$ High resolution spectra show that this line is mainly due to [OIII].

${ }^{\natural}$ Probably saturated.

* Measured from the short exposure to determine the $N_{\mathrm{e}}$.

\section{The spectrum of NGC 6741}

NGC 6741 is a small nebula, not more than $9^{\prime \prime}$ in diameter. This means that in well centered ISO and IUE measurements, the entire intensity of the nebula is observed. This makes it easier to compare the various wavelength regions. 
On the other hand, radio observations of the nebula are made somewhat more difficult by the fact that there is another strong source only $2^{\prime}$ away (see Condon et al. 1998). This makes the several single dish observations useless for determining the flux density of the nebula. There are several VLA observations which can be used. Condon et al. (1998) measure $132.8 \mathrm{mJy}$ at $21 \mathrm{~cm}$, which agrees with the $21 \mathrm{~cm}$ measurement of Phillips \& Mampaso (1988). However a small nebula could be partly optically deep at this wavelength and the flux density would be too small. Phillips \& Mampaso (1988) find a flux density of $127 \mathrm{mJy}$ also at $2 \mathrm{~cm}$, which is equivalent to $144 \mathrm{mJy}$ at $6 \mathrm{~cm}$. Isaacman (1984) measures $153 \mathrm{mJy}$ at $6 \mathrm{~cm}$. We shall use $150 \mathrm{mJy}$ at this wavelength.

\subsection{ISO observations}

The ISO SWS observations were made with both the SWS01 and SWS02 observing templates. They were made with a position centered at $\operatorname{RA}(2000) 19^{\mathrm{h}} 02^{\mathrm{m}} 37.0^{\mathrm{s}}$ and $\operatorname{Dec}(2000)-00^{\circ} 26^{\prime} 56.0^{\prime \prime}$. The diaphragm was the same as described for NGC 7662. The nebula is small enough so that the entire nebula has been measured. The intensities of the various lines are shown in Table 5. Not all the lines were observed by ISO. The [SIV] and [Ar III] lines have been taken from ground based measurements by Beck et al. (1981) and Grasdalen (1979). Beck et al. use a $7^{\prime \prime}$ aperture which is just sufficient to measure the entire nebula. Grasdalen uses an $11^{\prime \prime}$ aperture which certainly measured the entire nebula. His values are slightly higher than those of Beck et al., and the table gives an average of both measurements. The data from the LWS spectrum are taken from Liu et al. (2001), and are taken with a $70^{\prime \prime}$ aperture which was large enough to measure the entire nebula, but small enough to avoid the nearby emission features. The uncertainty in the SWS observations is estimated to be $10 \%$.

Table 5. ISO observations of NGC6741 (in units of $10^{-12} \mathrm{erg} \mathrm{cm}^{-2} \mathrm{~s}^{-1}$ ).

\begin{tabular}{lrr|lrr}
\hline Ident. & $\lambda(\mu \mathrm{m})$ & Intens. & Ident. & $\lambda(\mu \mathrm{m})$ & Intens. \\
\hline H I 5-4 & 4.052 & 2.74 & {$[\mathrm{Ne} \mathrm{V}]$} & 24.31 & 21.8 \\
{$[\mathrm{Mg} \mathrm{IV}]$} & 4.487 & 1.98 & {$[\mathrm{O}$ IV] } & 25.89 & 81.9 \\
{$[\mathrm{Ar}$ VI] } & 4.530 & 1.14 & {$[\mathrm{Ne} \mathrm{III}]$} & 36.01 & 6.99 \\
{$[\mathrm{Mg} \mathrm{V}]$} & 5.609 & 1.71 & {$[\mathrm{~N} \mathrm{III}]$} & 57 & 7 \\
{$[\mathrm{Ne} \mathrm{II}]$} & 12.81 & 5.34 & {$[\mathrm{O}$ III] } & 52 & 26 \\
{$[\mathrm{Ne} \mathrm{V}]$} & 14.32 & 23.8 & {$[\mathrm{O}$ III] } & 88 & 5.4 \\
{$[\mathrm{Ne}$ III $]$} & 15.55 & 76.5 & {$[\mathrm{~S} \mathrm{IV}]$} & 10.5 & 21 \\
\hline
\end{tabular}

\subsection{Extinction}

The $6 \mathrm{~cm}$ radio flux density of $150 \mathrm{mJy}$ implies that

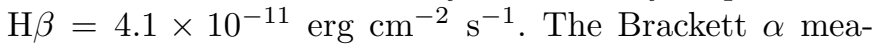
surement of $2.90 \times 10^{-12}$ (after a small extinction correction) predicts an $\mathrm{H} \beta$ intensity of $4.0 \times 10^{-11} \mathrm{erg} \mathrm{cm}^{-2} \mathrm{~s}^{-1}$ if $T_{\mathrm{e}}=12500 \mathrm{~K}$. The observed $\mathrm{H} \beta=4.36 \times$ $10^{-12} \mathrm{erg} \mathrm{cm}^{-2} \mathrm{~s}^{-1}$. The leads to a value of extinction about $c=1.0$. From the Balmer dectrement, Hyung \& Aller (1997b) find a value of $c=1.1$.

The correction for extinction was made in the following way. For the infrared lines in Table 5, a correction based on $c=1.1$ was used. Only a minor difference occurs if $c=1.0$ is used. For the visible lines, $c=1.1$ is used to obtain all lines with respect to $\mathrm{H} \beta$. For $\mathrm{H} \beta$ itself a value of $4.1 \times 10^{-11} \mathrm{erg} \mathrm{cm}^{-2} \mathrm{~s}^{-1}$ is used. Using the value of the He II $\lambda 4868 \AA$ line found in this way, a value of the He II $\lambda 1640 \AA$ line is found from the prediction of Hummer \& Storey (1987) at $T_{\mathrm{e}}=15000 \mathrm{~K}$. The IUE ultraviolet observations are then multiplied by a factor which gives the predicted $\lambda 1640 \AA$ line intensity, which takes care of the largest part of the extinction correction. The ratio of other ultraviolet lines to $\lambda 1640 \AA$ is then made using $c=1.1\left(E_{B-V}=0.75\right)$. The extinction curve of Fluks et al. (1994) is used throughout.

\subsection{The visual and the ultraviolet spectrum}

The visual spectrum used is that of Hyung \& Aller (1997b). As before, the spectrum refers to a $6^{\prime \prime} \times 4^{\prime \prime}$ region in the nebula. Since NGC 6741 is only slightly larger than this, it is probably a good approximation to assume that this represents the entire visual spectrum of the nebula. After correcting for extinction with respect to $\mathrm{H} \beta$, the spectrum is normalized to $\mathrm{H} \beta=4.1 \times 10^{-11} \mathrm{erg} \mathrm{cm}^{-2} \mathrm{~s}^{-1}$.

The $[\mathrm{NeV}]$ line at $\lambda 3426 \AA$ measured by Rowlands et al. (1984) was used $\left(0.7 \times 10^{-11} \mathrm{erg} \mathrm{cm}^{-2} \mathrm{~s}^{-1}\right.$ before correction for extinction).

The IUE spectrum is taken from NEWSIPS processed SWP 08970 and LWR 07721 spectra. Both are low resolution spectra, and both have an exposure of $240 \mathrm{~min}$. They are similar, but probably more accurate than the values given by Hyung \& Aller (1997b). The measured intensities are shown in the third column of Table 6 , and corrected for extinction in the fourth column of the table.

\section{Chemical composition of the nebulae}

The method of analysis is the same as used in the papers cited in the introduction. First the electron density and temperature as a function of the ionization potential is determined. Then the ionic abundances are determined, using density and temperature appropriate for the ion under consideration, together with Eq. (1). Then the element abundances are found for those elements in which a sufficient number of ions abundances have been determined.

\subsection{Electron density}

The ions used to determine $N_{\mathrm{e}}$ are listed in the first column of Table 7 . The ionization potential required to reach that ionization stage, and the wavelengths of the lines used, are given in Cols. 2 and 3. Note that the wavelength units are $\AA$ when 4 ciphers are given and microns when 
Table 6. IUE spectrum of NGC 6741.

\begin{tabular}{|c|c|c|c|}
\hline $\begin{array}{l}\lambda \\
(\AA)\end{array}$ & Ion & $\begin{array}{l}\text { Measured } \\
\text { Intens. }^{1}\end{array}$ & $\begin{array}{c}\text { Corrected } \\
\text { Intens. }^{2}\end{array}$ \\
\hline 1240 & $\mathrm{Nv}$ & 4.1 & 4.3 \\
\hline 1279.54 & & 3.6 & 2.9 \\
\hline 1288.31 & & 1.8 & 1.45 \\
\hline 1311.03 & & 1.7 & 1.14 \\
\hline 1400 & O IV] & 7.8 & 3.40 \\
\hline 1434.63 & & 2.3 & 0.90 \\
\hline 1487.01 & N IV] & 3.6 & 1.29 \\
\hline 1549.6 & $\mathrm{C}$ IV & 64.4 & 22.7 \\
\hline 1615.09 & & 3.5 & 1.2 \\
\hline 1641.50 & He II & 54.3 & 17.9 \\
\hline 1664.27 & O III] & 7.7 & 2.48 \\
\hline 1731.56 & & 2.0 & 0.58 \\
\hline 1751.59 & $\mathrm{~N}$ II] & 13.7 & 3.77 \\
\hline 1813.91 & & 1.3 & 0.4 \\
\hline 1834.46 & & 2.3 & 0.7 \\
\hline 1888.23 & Si III] & 2.6: & $0.8:$ \\
\hline 1908.54 & C III] & 89.4 & 29.3 \\
\hline 2030.93 & & 6.6 & 2.3 \\
\hline 2328.92 & C II] & 15.8 & 7.94 \\
\hline 2425.53 & {$[\mathrm{NeIV}]$} & 29.5 & 8.20 \\
\hline 2472.08 & {$[\mathrm{O}$ II $]$} & 8.9 & 1.97 \\
\hline 2734.55 & He II & 4.6 & 0.43 \\
\hline 2786.52 & {$[\mathrm{Mg} \mathrm{V}]$} & 5.8: & $0.52:$ \\
\hline 2799.47 & $\mathrm{Mg}$ II & 18.8 & 1.61 \\
\hline 2836.68 & O III & 6.8 & 0.52 \\
\hline 3024.70 & O III & 4.2 & 0.24 \\
\hline 3047.16 & O III & 12.3 & 0.70 \\
\hline 3133.99 & O III & 85.6 & 4.35 \\
\hline 3202.50 & He II & 21.5 & 1.02 \\
\hline
\end{tabular}

3 ciphers are shown. The observed ratio of the lines is given in the fourth and sixth column for NGC 7662 and NGC 6741 respectively. The corresponding electron density is given in the fifth and seventh column. The temperature used is discussed in the following section, but is unimportant since these line ratios are essentially determined by the density. There are not so many density determinations for NGC 6741 because (1) no high resolution IUE measurements have been made, and (2) complete ISO scan has not been made.

There is no indication that the electron density varies with ionization potential in a systematic way. As already pointed out by Liu et al. (2001) the [O III] lines always give a lower density than the other lines. Ignoring these lines, the electron density appears to be about $3500 \mathrm{~cm}^{-3}$ for NGC 7662 and about $7500 \mathrm{~cm}^{-3}$ for NGC 6741 .

It is interesting to compare this value of the density with the rms density found from the $\mathrm{H} \beta$ line. This depends on the distance of the nebula which isn't accurately known, and on the angular size of the nebula. For this calculation we shall use a distance of $0.8 \mathrm{kpc}$ for NGC 7662
(Hajian \& Terzian 1996) and $1.5 \mathrm{kpc}$ for NGC 6741 (Acker et al. 1992). The volume of each nebula is represented by a sphere of $15^{\prime \prime}$ and $3.9^{\prime \prime}$ respectively. The $\mathrm{H} \beta$ flux has been given above and the electron temperature will be discussed below. We obtain a value of $2300 \mathrm{~cm}^{-3}$ for NGC 7662 and $6500 \mathrm{~cm}^{-3}$ for NGC 6741 . These values are uncertain because the distance is not well known. However the rms density varies only as the square root of the distance. It is therefore likely that the similarity of these values to the forbidden line densities is real. This probably indicates inhomogeneities do not play a dominant role in determining the density in either nebula. We will use the forbidden line densities in further discussion of the abundances.

The fact that the $[\mathrm{NeV}] 24.3 / 14.3$ line ratio is "impossible" for any density deserves some comment. Rubin et al. (Mexico City conference of Nov. 2000) have studied this line ratio in several $\mathrm{PN}$ and have found other cases when this is also true. They suspect that this may be due to errors in the atomic parameters involved in these transitions.

\subsection{Electron temperature}

A number of ions have lines originating from energy levels far enough apart that their ratio is sensitive to the temperature. These are listed in Table 8, which is arranged similarly to the previous table. It is clear that the electron temperature increases for the higher ionization potentials. This is the same general effect which has been found in the other nebulae with ISO observations discussed previously. In the previous nebulae the temperature found from the [Ne III] lines is lower than what would be expected from the ions with lower and higher ionization potential. This may also be the case for NGC 7662 and NGC 6741, but it is not so large an effect as in the other nebulae. Still, we give less weight to this ion in defining the temperature vs. ionization curve, which is used to give the temperature for individual ions. Thus for each nebula a diagram was made plotting the electron temperature against ionization potential for each ion listed in Table 8 (except [Ne III]). The plots are shown as Figs. 1 and 2. In these figures the best fitting line is drawn and it is this line that is used to assign a temperature to a given ion to determine its abundance.

\subsection{Ionic and element abundances}

The ionic abundances have been determined using the following equation:

$\frac{N_{\text {ion }}}{N_{\mathrm{p}}}=\frac{I_{\text {ion }}}{I_{\mathrm{H}_{\beta}}} N_{\mathrm{e}} \frac{\lambda_{\mathrm{ul}}}{\lambda_{\mathrm{H}_{\beta}}} \frac{\alpha_{\mathrm{H}_{\beta}}}{A_{\mathrm{ul}}}\left(\frac{N_{\mathrm{u}}}{N_{\text {ion }}}\right)^{-1}$

where $I_{\mathrm{ion}} / I_{\mathrm{H} \beta}$ is the measured intensity of the ionic line compared to $\mathrm{H} \beta, N_{\mathrm{p}}$ is the density of ionized hydrogen, $\lambda_{\mathrm{ul}}$ is the wavelength of this line, $\lambda_{\mathrm{H} \beta}$ is the wavelength of $\mathrm{H} \beta$, $\alpha_{\mathrm{H} \beta}$ is the effective recombination coefficient for $\mathrm{H} \beta, A_{\mathrm{ul}}$ is the Einstein spontaneous transition rate for the line, 
Table 7. Electron density indicators.

\begin{tabular}{lccccccc}
\hline & & & \multicolumn{2}{c}{ NGC 7662 } & & \multicolumn{2}{c}{ NGC 6741 } \\
Ion & $\begin{array}{c}\text { Ioniz. } \\
\text { Pot. }(\mathrm{eV})\end{array}$ & $\begin{array}{c}\text { Lines } \\
\text { Used }\end{array}$ & $\begin{array}{c}\text { Observed } \\
\text { Ratio }\end{array}$ & $\begin{array}{c}N_{\mathrm{e}} \\
\left(\mathrm{cm}^{-3}\right)\end{array}$ & & $\begin{array}{c}\text { Observed } \\
\text { Ratio }\end{array}$ & $\begin{array}{c}N_{\mathrm{e}} \\
\left(\mathrm{cm}^{-3}\right)\end{array}$ \\
\hline S II & 10.4 & $6731 / 6716$ & 1.60 & 3200 & & 1.81 & 6000 \\
O II & 13.3 & $3726 / 3729$ & 1.92 & 5000 & & 2.15 & 8500 \\
S III & 23.3 & $18.7 / 33.4$ & 2.48 & 3800 & & \\
Cl III & 23.8 & $5538 / 5518$ & 1.24 & 4500 & & 1.61 & 9000 \\
C III & 24.4 & $1907 / 1909$ & 1.34 & 5000 & & & \\
N III & 29.6 & $1752 / 1749.7$ & 0.66 & 3200 & & & \\
O III & 35.1 & $52 / 88$ & 2.63 & 1000 & & 4.82 & 3000 \\
Ar IV & 40.7 & $4740 / 4711$ & 0.98 & 3000 & & 1.19 & 5500 \\
Ne III & 41.0 & $15.5 / 36.0$ & 9.07 & $1000:$ & & & \\
Ne IV & 63.5 & $2425 / 2422$ & 1.13 & 3200 & & & \\
Ne V & 97.1 & $24.3 / 14.3$ & 1.10 & Imposs. & & \\
\hline
\end{tabular}

Table 8. Electron temperature indicators.

\begin{tabular}{|c|c|c|c|c|c|c|}
\hline \multirow[b]{2}{*}{ Ion } & \multirow[b]{2}{*}{$\begin{array}{c}\text { Ioniz. } \\
\text { Pot. }(\mathrm{eV})\end{array}$} & \multirow[b]{2}{*}{$\begin{array}{l}\text { Lines } \\
\text { Used }\end{array}$} & \multicolumn{2}{|c|}{ NGC 7662} & \multicolumn{2}{|c|}{ NGC 6741} \\
\hline & & & $\begin{array}{c}\text { Observed } \\
\text { Ratio }\end{array}$ & $\begin{array}{c}T_{\mathrm{e}} \\
(\mathrm{K})\end{array}$ & $\begin{array}{c}\text { Observed } \\
\text { Ratio }\end{array}$ & $\begin{array}{l}T_{\mathrm{e}} \\
(\mathrm{K})\end{array}$ \\
\hline O II & 13.6 & $7320 / 3727$ & 0.49 & 11800 & uncert. & \\
\hline N II & 14.5 & $5755 / 6584$ & 0.042 & 14500 & 0.0216 & 10300 \\
\hline S III & 23.3 & $6312 / 18.7$ & 0.057 & 10800 & & \\
\hline Ar III & 27.6 & $5192 / 8.99$ & 0.0156 & 11900 & 0.020 & 12500 \\
\hline O III & 35.1 & $4363 / 5007$ & 0.0133 & 12800 & 0.0132 & 12600 \\
\hline Ne III & 41.0 & $3868 / 15.5$ & 1.23 & 12200 & 1.27 & 12500 \\
\hline O IV & 54.9 & $1400 / 25.9$ & 0.059 & 14700 & 0.274 & 17000 \\
\hline $\mathrm{Ne}$ IV & 63.5 & $2425 / 4725$ & 84.7 & 19500 & 81.3 & 18300 \\
\hline $\mathrm{Ne} V$ & 97.1 & $3425 / 14.3$ & 0.518 & 18300 & 0.88 & 21000 \\
\hline $\mathrm{Mg} \mathrm{V}$ & 109 & $2783 / 5.61$ & 0.563 & 17000 & uncert. & \\
\hline
\end{tabular}

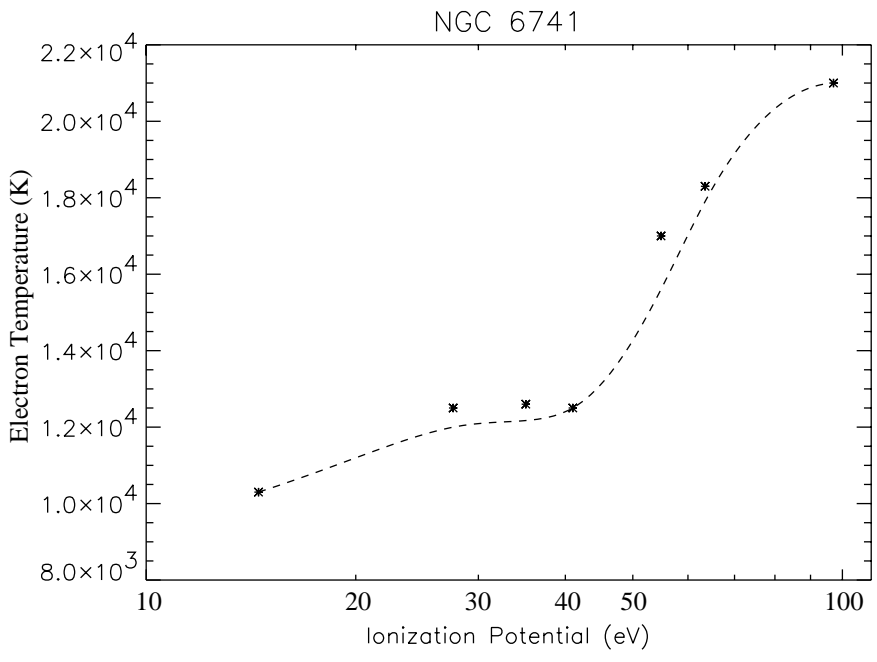

Fig. 1. The effective electron temperature is plotted against the ionization potential for the ions listed in Table 8 for NGC 6741. The $x$-axis is in logarithmic scale.

and $N_{\mathrm{u}} / N_{\text {ion }}$ is the ratio of the population of the level from which the line originates to the total population of the ion. This ratio has been determined using a five level atom.

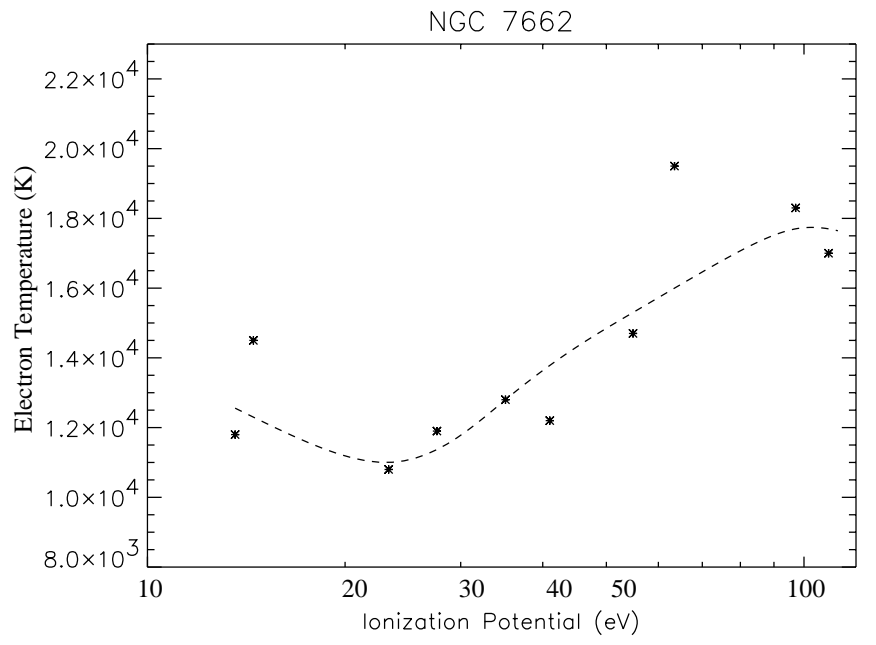

Fig. 2. The effective electron temperature is plotted against the ionization potential for the ions listed in Table 8 for NGC 7662. The $x$-axis is in logarithmic scale.

\subsubsection{NGC 7662}

The results are given in Table 9 , where the first column lists the ion concerned, and the second column the line used for the abundance determination. Sometimes more 
Table 9. Ionic concentrations and chemical abundances in NGC 7662. Wavelength in Angstrom for all values of $\lambda$ above 1000, otherwise in $\mu \mathrm{m}$.

\begin{tabular}{lccccc}
\hline $\mathrm{Ion}$ & $\lambda$ & $n_{\text {ion }} / n_{\mathrm{p}}$ & $\Sigma n_{\text {ion }} / n_{\mathrm{p}}$ & $\mathrm{ICF}$ & $n_{\mathrm{el} .} / n_{\mathrm{p}}$ \\
\hline $\mathrm{He}^{+}$ & 5875 & 0.030 & & & \\
$\mathrm{He}^{++}$ & 4686 & 0.058 & 0.088 & & 0.088 \\
$\mathrm{C}^{+}$ & 2325 & $2.3(-6)$ & & & \\
$\mathrm{C}^{++}$ & 1909 & $2.0(-4)$ & & & \\
$\mathrm{C}^{+3}$ & 1548 & $1.0(-4)$ & $3.0(-4)$ & 1.2 & $3.6(-4)$ \\
$\mathrm{N}^{+}$ & 6584 & $2.6(-7)$ & & & \\
$\mathrm{N}^{++}$ & 1750,57 & $3.7(-5)$ & & & \\
$\mathrm{N}^{+3}$ & 1487 & $2.6(-5)$ & & & \\
$\mathrm{N}^{+4}$ & 1238 & $3.2(-6)$ & $6.6(-5)$ & $1.0:$ & $6.7(-5)$ \\
$\mathrm{O}^{+}$ & 3727 & $2.8(-6)$ & & & \\
$\mathrm{O}^{++}$ & 5007,52 & $2.5(-4)$ & & & \\
$\mathrm{O}^{+3}$ & $1400,25.8$ & $1.2(-4)$ & $3.8(-4)$ & 1.1 & $4.2(-4)$ \\
$\mathrm{Ne}^{+}$ & 12.8 & $1.6(-5)$ & & & \\
$\mathrm{Ne}^{++}$ & 15.5 & $3.4(-5)$ & & & \\
$\mathrm{Ne}^{+3}$ & 2425 & $1.1(-5)$ & & & \\
$\mathrm{Ne}^{+4}$ & $14.3,24.3$ & $3.2(-6)$ & $6.2(-5)$ & 1.03 & $6.4(-5)$ \\
$\mathrm{Mg}^{+3}$ & 4.459 & $7.8(-6)$ & & & \\
$\mathrm{Mg}^{+4}$ & 5.606 & $6.5(-7)$ & $8.5(-6)$ & $2:$ & $1.7(-5)$ \\
$\mathrm{S}^{+}$ & 6731 & $1.8(-8)$ & & & \\
$\mathrm{S}^{++}$ & 18.7 & $1.2(-6)$ & & & \\
$\mathrm{S}^{+3}$ & 10.5 & $3.2(-6)$ & $4.4(-6)$ & 1.5 & $6.6(-6)$ \\
$\mathrm{Cl}^{++}$ & 5538 & $3.2(-8)$ & & & \\
$\mathrm{Cl}^{+3}$ & 8046 & $4.8(-8)$ & $8.0(-8)$ & 1.7 & $1.4(-7)$ \\
$\mathrm{Ar}^{++}$ & 8.99 & $5.7(-7)$ & & & \\
$\mathrm{Ar}^{+3}$ & 4741 & $7.8(-7)$ & & & \\
$\mathrm{Ar}^{+4}$ & 7.91 & $9.2(-8)$ & & & \\
$\mathrm{Ar}^{+5}$ & 4.54 & $2.6(-8)$ & $1.5(-6)$ & 1.4 & $2.1(-6)$ \\
$\mathrm{K}^{3+}$ & 6102 & $1.6(-8)$ & & & \\
\hline & & & & & \\
\hline
\end{tabular}

than one line was used. The third column gives the ionic abundance, while the fourth column gives the sum of the observed stages of ionization of a given element. The fifth column gives the Ionization Correction Factor (ICF). This has been determined both empirically and with the help of model nebulae. Notice that the ICF is usually small, less than a factor 1.5, and the element abundances, given in the last column, are probably well determined. The abundance of $\mathrm{Mg}$ is somewhat less certain, but probably correct to within $50 \%$.

\subsubsection{NGC 6741}

The results are given in Table 10, where the columns are the same as for NGC 7662. The degree of ionization is similar in both nebulae. The He abundance is somewhat higher in NGC 6741, and all other elements are about a factor of two higher. The single exception to this is $\mathrm{Mg}$, which is about a factor of 6 higher in NGC 7662. But the abundance of $\mathrm{Mg}$ in NGC 7662 is very similar to that in NGC 7027 or NGC 6302 and within a factor of two of the
Table 10. Ionic concentrations and chemical abundances in NGC 6741. Wavelength in Angstrom for all values of $\lambda$ above 1000 , otherwise in $\mu \mathrm{m}$.

\begin{tabular}{|c|c|c|c|c|c|}
\hline Ion & $\lambda$ & $n_{\text {ion }} / n_{\mathrm{p}}$ & $\Sigma n_{\text {ion }} / n_{\mathrm{p}}$ & $\mathrm{ICF}$ & $n_{\mathrm{el} .} / n_{\mathrm{p}}$ \\
\hline $\mathrm{He}^{+}$ & 5875 & 0.070 & & & \\
\hline $\mathrm{He}^{++}$ & 4686 & 0.041 & 0.111 & & 0.111 \\
\hline $\mathrm{C}^{+}$ & 2325 & $3.5(-5)$ & & & \\
\hline $\mathrm{C}^{++}$ & 1909 & $4.8(-4)$ & & & \\
\hline $\mathrm{C}^{+3}$ & 1548 & $5.7(-5)$ & $5.8(-4)$ & 1.1 & $6.4(-4)$ \\
\hline $\mathrm{N}^{+}$ & 6584 & $4.8(-5)$ & & & \\
\hline $\mathrm{N}^{++}$ & 1750,57 & $1.8(-4)$ & & & \\
\hline $\mathrm{N}^{+3}$ & 1487 & $2.3(-5)$ & & & \\
\hline $\mathrm{N}^{+4}$ & 1238 & $2.1(-5)$ & $2.7(-4)$ & 1.0 & $2.8(-4)$ \\
\hline $\mathrm{O}^{+}$ & 3727 & $1.1(-4)$ & & & \\
\hline $\mathrm{O}^{++}$ & 5007,52 & $3.2(-4)$ & & & \\
\hline $\mathrm{O}^{+3}$ & $1400,25.9$ & $1.7(-4)$ & $6.0(-4)$ & 1.1 & $6.6(-4)$ \\
\hline $\mathrm{Ne}^{+}$ & 12.8 & $1.5(-5)$ & & & \\
\hline $\mathrm{Ne}^{++}$ & 15.5 & $1.2(-4)$ & & & \\
\hline $\mathrm{Ne}^{+3}$ & 2425 & $3.3(-5)$ & & & \\
\hline $\mathrm{Ne}^{+4}$ & $14.3,24.3$ & $1.0(-5)$ & $1.8(-4)$ & 1.03 & $1.8(-4)$ \\
\hline $\mathrm{Mg}^{+3}$ & 4.459 & $3.5(-7)$ & & & \\
\hline $\mathrm{Mg}^{+4}$ & 5.606 & $9.8(-7)$ & $1.3(-6)$ & 2: & $2.7(-6)$ \\
\hline $\mathrm{S}^{+}$ & 6731 & $1.5(-6)$ & & & \\
\hline $\mathrm{S}^{++}$ & 18.7 & $3.3(-6)$ & & & \\
\hline $\mathrm{S}^{+3}$ & 10.5 & $2.5(-6)$ & $7.3(-6)$ & 1.5 & $1.1(-5)$ \\
\hline $\mathrm{Cl}^{+}$ & 8578 & $4.1(-8)$ & & & \\
\hline $\mathrm{Cl}^{++}$ & 5538 & $8.1(-8)$ & & & \\
\hline $\mathrm{Cl}^{+3}$ & 8046 & $2.5(-8)$ & $1.5(-7)$ & 1.6 & $2.4(-7)$ \\
\hline $\mathrm{Ar}^{++}$ & 8.99 & $2.5(-6)$ & & & \\
\hline $\mathrm{Ar}^{+3}$ & 4741 & $8.0(-7)$ & & & \\
\hline $\mathrm{Ar}^{+4}$ & 7007 & $2.0(-7)$ & & & \\
\hline $\mathrm{Ar}^{+5}$ & 4.54 & $3.4(-8)$ & $3.5(-6)$ & 1.4 & $4.9(-6)$ \\
\hline
\end{tabular}

solar abundance. The low value of $\mathrm{Mg}$ in NGC 6741 is thus anomalous. It could be that much of the $\mathrm{Mg}$ is trapped in dust grains in this nebula, but the question then arises as to why this does not happen in the other nebulae.

\section{Comparison with other abundance determinations}

\subsection{NGC 7662}

Table 11 shows a comparison of our abundances with the most important determinations in the past 20 years. There is reasonable agreement to within a factor of two. Hyung \& Aller (1997a) have made two determinations of the abundances. The first method is to determine the individual ionic abundances and to sum these to get the element abundance. This is listed as " $\Sigma_{\text {ion }}$ " in the table. Further, they have constructed different model nebulae and compared the resultant line flux with that observed. The abundances were changed until a best fit was obtained. This is listed as "Model" in the table. As can be seen, the difference between these two determinations is 
Table 11. Comparison of abundances in NGC 7662.

\begin{tabular}{lrrrrr}
\hline \multirow{2}{*}{ Elem. } & \multirow{2}{*}{ Present } & \multicolumn{2}{c}{$\mathrm{HA}(1)$} & $\mathrm{B}(2)$ & $\mathrm{H}(3)$ \\
\cline { 3 - 4 } & Abund. & Model & $\Sigma_{\text {ion }}$ & & \\
\hline $\mathrm{He}$ & 0.088 & 0.093 & 0.093 & 0.093 & 0.093 \\
$\mathrm{C}$ & $3.6(-4)$ & $4.5(-4)$ & $9.5(-4)$ & $6.8(-4)$ & $6.2(-4)$ \\
$\mathrm{N}$ & $6.7(-5)$ & $4.7(-5)$ & $15.4(-5)$ & $11.0(-5)$ & $6.0(-5)$ \\
$\mathrm{O}$ & $4.2(-4)$ & $3.5(-4)$ & $7.0(-4)$ & $4.3(-4)$ & $3.6(-4)$ \\
$\mathrm{S}$ & $6.6(-6)$ & $7.0(-6)$ & $4.7(-6)$ & $4.2(-6)$ & $1.5(-6)$ \\
$\mathrm{Ar}$ & $2.1(-6)$ & $2.0(-6)$ & $1.3(-6)$ & $1.5(-6)$ & \\
$\mathrm{Ne}$ & $6.4(-5)$ & $6.3(-5)$ & $9.2(-5)$ & $9.1(-5)$ & $7.0(-5)$ \\
\hline
\end{tabular}

(1) Hyung \& Aller (1997a); (2) Barker (1986); (3) Harrington et al. (1982).

rather large. Our abundance determination, which is made by summing the individual ions, is actually in good agreement with their "model" determination.

In spite of the comparatively small differences among the various determinations, the present determination indicates that the nebula is oxygen rich (the $\mathrm{C} / \mathrm{O}$ ratio is less than unity). Earlier results indicated the opposite. But the differences from unity are still marginal.

The helium abundance differs slightly with the earlier determinations. This is due to the more recent determination of the He I spectrum by Benjamin et al. (1999). In addition only the $\lambda 5875 \AA$ line was used, because we feel that the theoretical determination of this line is the most reliable.

\subsection{NGC 6741}

The same comparison for NGC 6741 is shown in Table 12. Many of the same comments as above apply also to this nebula. Hyung \& Aller (1997b) have also given two abundance determinations, one using a model and the other by summing the individual ions observed. This time the two results are somewhat closer than before, although there is almost a factor of two difference in the two nitrogen determinations. Our results this time are closer to their results summing the individual ions of each element.

Interestingly our results question the previous determination that the nebula is carbon rich. The present results indicate that oxygen and carbon are equally abundant in this nebula.

\subsection{Errors}

In both this nebula and in NGC 7662 it would be interesting to determine the errors in the abundance determination. Unfortunately this is difficult to do. The reason for this is the following. The error can occur at several stages in the determination. An error can occur in the intensity determination and this can be specified: it is probably less
Table 12. Comparison of abundances in NGC 6741.

\begin{tabular}{|c|c|c|c|c|c|}
\hline \multirow[t]{2}{*}{ Elem. } & \multirow{2}{*}{$\begin{array}{l}\text { Present } \\
\text { Abund. }\end{array}$} & \multicolumn{2}{|c|}{$\mathrm{HA}(1)$} & \multirow[t]{2}{*}{$\mathrm{KB}(2)$} & \multirow[t]{2}{*}{$\mathrm{A}(3)$} \\
\hline & & Model & $\Sigma_{\text {ion }}$ & & \\
\hline $\mathrm{He}$ & 0.11 & 0.11 & 0.11 & 0.11 & 0.11 \\
\hline $\mathrm{C}$ & $6.4(-4)$ & $8.0(-4)$ & $7.3(-4)$ & $6.5(-4)$ & $12.0(-4)$ \\
\hline $\mathrm{N}$ & $2.8(-4)$ & $1.4(-4)$ & $2.4(-4)$ & $1.4(-4)$ & $5.2(-4)$ \\
\hline $\mathrm{O}$ & $6.6(-4)$ & $4.5(-4)$ & $5.4(-4)$ & $4.9(-4)$ & $5.4(-4)$ \\
\hline S & $1.1(-5)$ & $5.7(-6)$ & $8.0(-6)$ & $8.1(-6)$ & $3.1(-6)$ \\
\hline $\mathrm{Ar}$ & $4.9(-6)$ & $3.5(-6)$ & $3.1(-6)$ & $2.4(-6)$ & $3.6(-6)$ \\
\hline $\mathrm{Ne}$ & $1.8(-4)$ & $1.0(-4)$ & $1.3(-4)$ & $1.3(-4)$ & $1.6(-4)$ \\
\hline
\end{tabular}

(1) Hyung \& Aller (1997b); (2) Kingsburgh \& Barlow (1994); (3) Aller et al. (1985).

than 30\% and may be lower for the stronger lines. An error may occur in correcting for the extinction, either because the extinction is incorrect or the average reddening law is not applicable. We have tried to minimize this possibility by making use of known atomic constants to relate the various parts of the spectrum. Thus the ratio of $\mathrm{Br} \alpha$ to $\mathrm{H} \beta$ is an atomic constant, as is the ratio of He II $\lambda 4686 \AA$ to $\lambda 1640 \AA$. The ratio of the infrared spectrum to the visible spectrum to the ultraviolet spectrum is fixed in this way, at least for the nebulae which are small enough to emit entirely in the diaphragm used. But if this is not the case, an error is introduced. The magnitude of this error is not possible to specify.

A further error is introduced by the correction for unseen stages of ionization. This varies with the element, but is usually small because very many ionization stages are observed. Thus for neon all but neutral neon is observed, so that the error will be small. For sulfur, on the other hand, the correction for SV and higher ionization stages is about $50 \%$, which could lead to greater uncertainties.

There is also an error due to uncertainties in the collisional cross-section and the transition probabilities. The former is estimated at $25 \%$ and the latter is smaller. One might think that when comparing nebulae this error will disappear, since the same error is made in all nebulae. This is partly true, but because the contribution of a given ion varies with the nebula, some unspecifiable error will remain.

To obtain some measure of the error we can look at the resulting sulfur abundances in the various nebulae shown in Table 13. This element is chosen because theoretical considerations predict that it will not be formed in the course of its evolution. In fact most of the nebulae have a sulfur abundance within $30 \%$ of the value $\mathrm{S} / \mathrm{H}=10^{-5}$ or slightly higher. Part of this could be due to differences in abundance at the time of formation of the star. This gives a justification for conducting the further discussion using a $30 \%$ error. 
Table 13. Comparison of abundances.

\begin{tabular}{lllllllllll}
\hline Elem. & NGC & He2-111 & NGC & NGC & NGC & NGC & NGC & NGC & Sun & Orion \\
& 6537 & & 6302 & 6445 & 6741 & 7027 & 7662 & $2440^{\dagger}$ & & \\
\hline $\mathrm{He}$ & 0.149 & 0.185 & 0.17 & $0.14:$ & 0.111 & 0.106 & 0.088 & 0.119 & 0.10 & 0.098 \\
$\mathrm{C}(-4)$ & 1.75 & 1.1 & 0.60 & 6.0 & 6.4 & 6.0 & 3.6 & 6.7 & 3.55 & 2.5 \\
$\mathrm{~N}(-4)$ & 4.5 & 3.0 & 2.9 & 2.4 & 2.8 & 1.6 & 0.67 & 4.4 & 0.93 & 0.60 \\
$\mathrm{O}(-4)$ & 1.85 & 2.7 & 2.3 & 7.4 & 6.6 & 4.1 & 4.2 & 3.6 & 7.4 & 4.4 \\
$\mathrm{Ne}(-4)$ & 1.7 & 1.6 & 2.2 & 2.0 & 1.8 & 1.0 & 0.64 & 1.1 & 1.2 & 0.78 \\
$\mathrm{Na}(-6)$ & 2.6 & & 2.6 & & & 1.2 & & & 2.0 & \\
$\mathrm{Mg}(-5)$ & 0.96 & $0.8:$ & 1.3 & $1.7:$ & 0.27 & 2.2 & 1.7 & & 3.8 & \\
$\mathrm{~S}(-5)$ & 1.1 & 1.5 & 0.78 & 0.79 & 1.1 & 0.94 & 0.88 & 0.47 & 1.86 & 1.5 \\
$\mathrm{Cl}(-7)$ & 2.4 & 3.5 & 3.4 & & 2,4 & 1.1 & 1.4 & & 1.9 & 2.2 \\
$\mathrm{Ar}(-6)$ & 4.1 & 5.5 & 6.0 & 3.8 & 4.9 & 2.3 & 2.1 & 3.1 & 3.6 & 6.3 \\
$\mathrm{C} / \mathrm{O}$ & 0.95 & 0.41 & 0.26 & 0.81 & 0.97 & 1.46 & 0.86 & 1.89 & 0.48 & 0.57 \\
$\mathrm{~N} / \mathrm{O}$ & 2.4 & 1.1 & 1.3 & 0.32 & 0.42 & 0.39 & 0.16 & 1.23 & 0.13 & 0.14 \\
$(\mathrm{C}+\mathrm{N}+\mathrm{O}) / \mathrm{H}^{*}$ & 8.1 & 6.8 & 5.8 & 15.8 & 15.8 & 11.7 & 8.5 & 14.7 & 11.9 & 7.5 \\
$(\mathrm{~N}+\mathrm{O}) / \mathrm{H}^{*}$ & 6.35 & 5.7 & 5.2 & 9.8 & 9.4 & 5.7 & 4.9 & 7.93 & 8.3 & 5.0 \\
\hline
\end{tabular}

* These ratios are in units of $10^{-4}$.

$\dagger$ Bernard Salas et al. (2002).

\section{Comparison of planetary nebula abundances}

In Table 13 we list the abundances of the seven PN which have been determined using ISO measurements. NGC 7662 and NGC 6741 are an interesting addition since they have a lower helium abundance than the others, closer to the solar helium abundance. The solar abundance is also shown in the table; it is taken from Anders \& Grevesse (1989) and Grevesse \& Noels (1993). The abundance in the Orion nebula in the last column is taken from Esteban et al. (1998).

In Fig. 3 the nitrogen abundance $\mathrm{N} / \mathrm{H}$ is plotted as a function of the helium abundance $\mathrm{He} / \mathrm{H}$. It is clear that the increase in the helium abundance by a factor of two is accompanied by an increase in the nitrogen abundance by a factor of 5 to 7 . The scatter is small enough to be caused by errors in the abundance determination. Presumably the nitrogen is being produced by the same process that produces the helium, and is being brought to the surface at the same time.

In Fig. 4 the carbon abundance $\mathrm{C} / \mathrm{H}$ is plotted as a function of the helium abundance $\mathrm{He} / \mathrm{H}$. The carbon abundance has a reasonably high value, about $5 \times 10^{-4}$, for low values of the helium abundance. This is similar to the solar carbon abundance. For the three nebulae with the highest helium abundance, the carbon abundance has fallen by a factor of 3 to 5 , indicating that the carbon is being converted to another element at these high helium abundances (presumably high initial mass stars). It appears that the scatter is somewhat higher for the carbon as for the nitrogen.

The behavior of the oxygen abundance $\mathrm{O} / \mathrm{H}$ is similar to that of carbon. For the same three nebula with the highest helium abundance, the oxygen abundance is

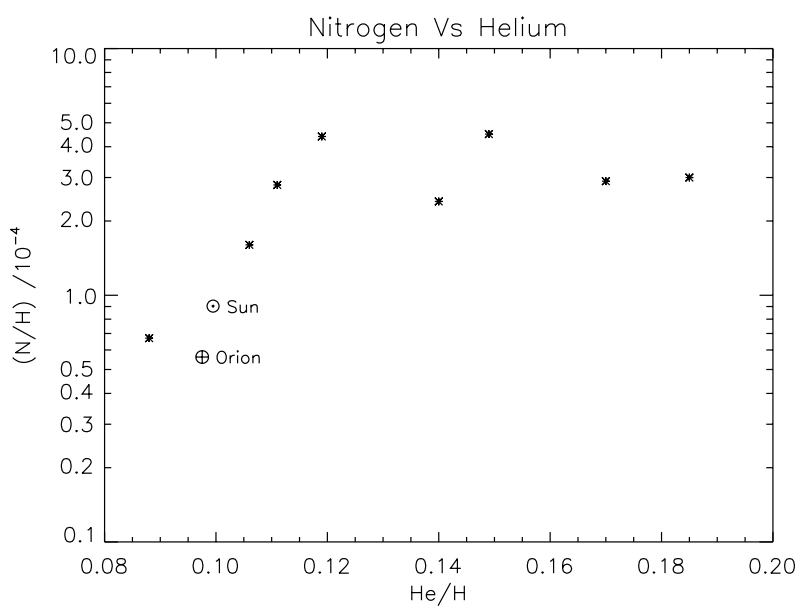

Fig. 3. The nitrogen abundance of the nebulae listed in Table 13 is plotted against their helium abundance. The solar value is shown as "Sun", while the values in the Orion nebula is indicated by a circle around a cross. The $y$-axis is in logarithmic scale. The nitrogen clearly increases as the helium increases.

clearly a factor of 2-3 lower, indicating that it too is being converted to another element. This other element must be mainly nitrogen, because, as can be seen in Table 13, the sum of carbon, nitrogen and oxygen is approximately constant for all nebulae. The sum of nitrogen and oxygen is also constant to within a factor of two. This constant is the same as shown by both the Sun and the Orion nebula. In Fig. 5 the nitrogen abundance is plotted against the oxygen abundance, showing an increase of the nitrogen abundance as the oxygen decreases. There is considerable scattering in the diagram however. Taken together, this qualitatively agrees with theoretical calculations of a third 


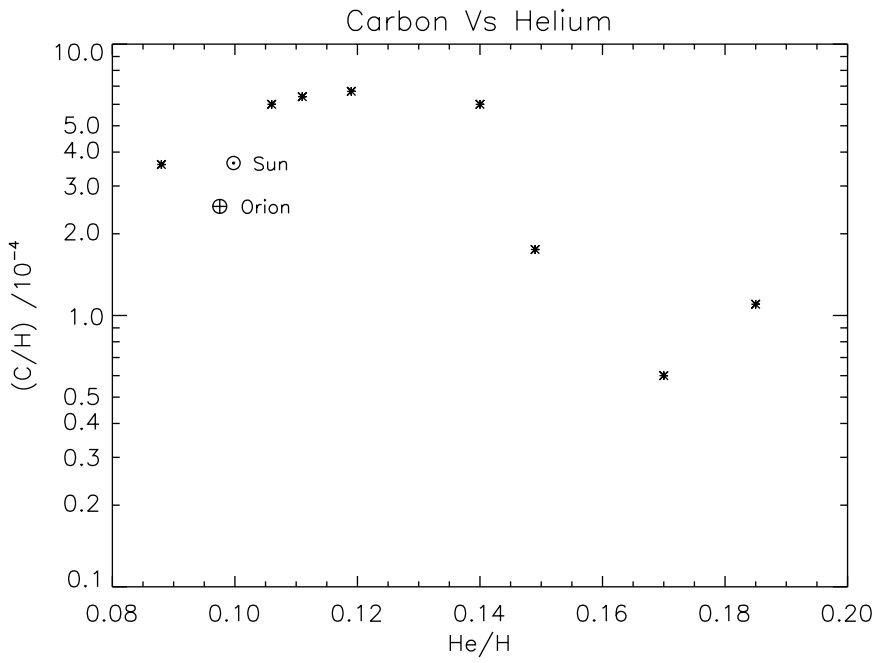

Fig. 4. The carbon abundance is plotted against the helium abundance for the same nebulae. The $y$-axis is in logarithmic scale. The decrease in carbon as the helium increases indicates its conversion to some other element in conditions which produce large amounts of helium.

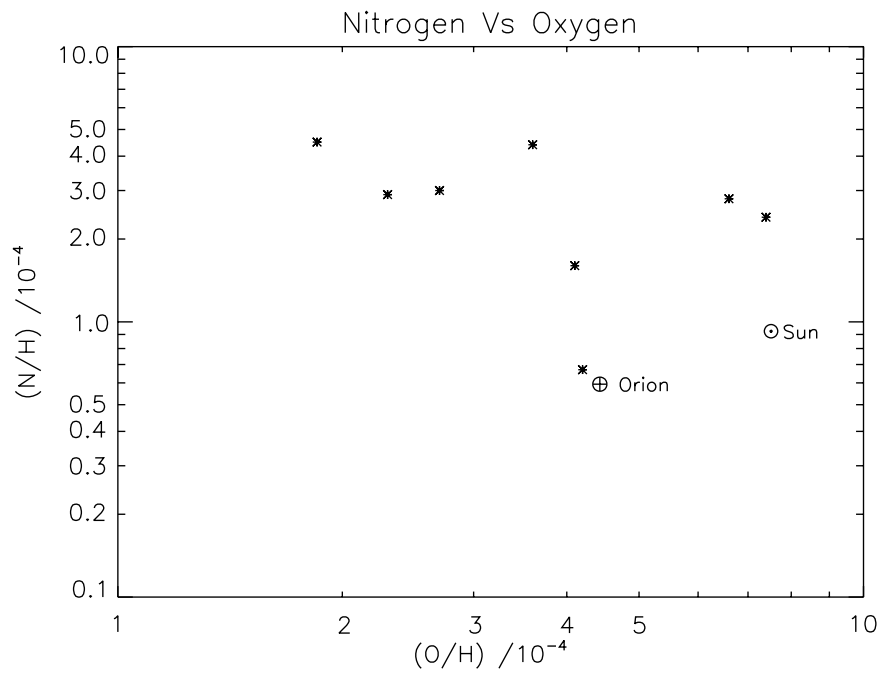

Fig. 5. The nitrogen abundance as a function of the oxygen abundance for the nebulae listed in Table 13. Both $x$ and $y$-axis are in logarithmic scale. It can be seen that the oxygen increase as the nitrogen decreases, but the scatter is large.

dredge-up event having occurred in NGC 6537, NGC 6302 and $\mathrm{He} 2-111$. But the large scatter may indicate that some other process is also occurring.

In Fig. 6 the neon abundance $\mathrm{Ne} / \mathrm{H}$ is plotted against the helium abundance. While the range is small, not more than a factor of three, there is a very good correlation with very little scatter. It may be that the neon abundance is better determined than other elements since all ionization stages are observed. The diagram makes it probable that neon is being created in higher mass stars. This has been suggested earlier (Corradi \& Schwarz 1995) on the basis of somewhat weaker evidence.

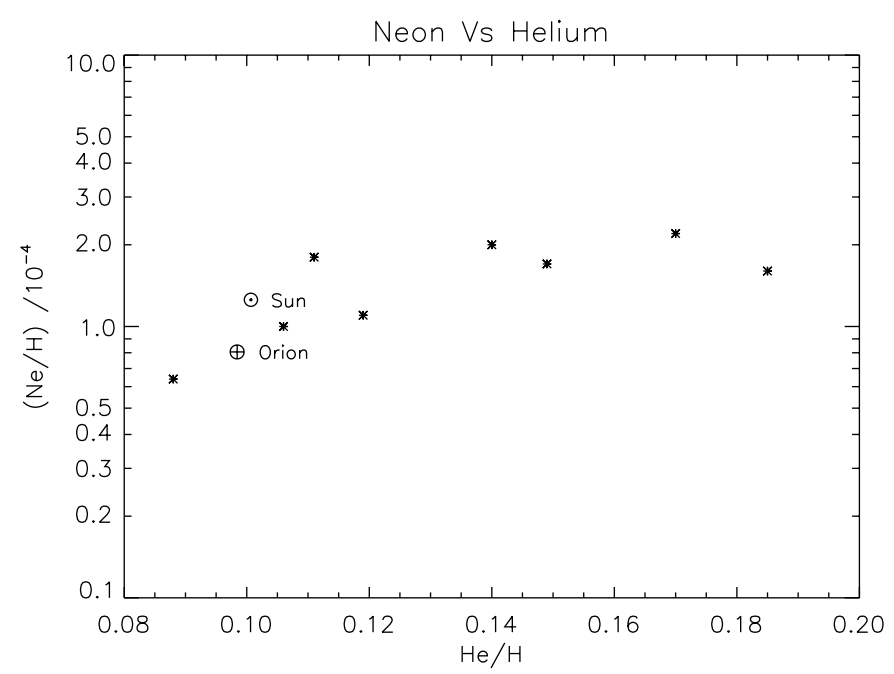

Fig. 6. The neon abundance is plotted against helium for the same nebulae. The $y$-axis is in logarithmic scale. The increased neon abundance argues for the production of some neon when a considerable amount of helium is produced.

The abundance of $\mathrm{Mg}$ has also been determined. The determination is made usually from $\mathrm{Mg}^{+3}$ and $\mathrm{Mg}^{+4}$, which probably represent about one half of the $\mathrm{Mg} . \mathrm{Mg}^{+}$ is usually also observed, but very little $\mathrm{Mg}$ is present in this ion. The $\mathrm{Mg}$ abundance is seen to be lower than solar, usually by a factor of 2 to 4 . It is generally thought that magnesium is a constituent of the nebular dust. In the two interstellar components seen in the direction of $\zeta \mathrm{Oph}$, the magnesium is depleted with respect to the sun by a factor of 35 (cold cloud) and 8 (warm cloud). This is considerably higher than found for the nebulae considered in Table 13. The single exception to this is NGC 6741, in which $\mathrm{Mg}$ is depleted by a factor of 14 . It would be interesting to see whether this correlates with some feature in the dust spectrum.

\section{References}

Acker, A., Ochsenbein, F., Stenholm, B., et al. 1992, Strasb.ESO Catalogue

Aller, L. H., \& Hyung, S. 1995, MNRAS, 276, 1101

Anders, E., \& Grevesse, N. 1989, Geochem. Cosmo., 53, 197

Barker, T. 1986, ApJ, 308, 314

Beck, S. C., Lacy, J. H., Townes, C. H., et al. 1981, ApJ, 249, 592

Benjamin, R. A., Skillman, E. D., \& Smits, D. P. 1999, ApJ, 514,307

Bernard Salas, J., Pottasch, S. R., Beintema, D. A., \& Wesselius, P. R. 2001, A\&A, 367, 949

Bernard Salas, J., Pottasch, S. R., Feibelman, W. A., \& Wesselius, P. R. 2002, A\&A, submitted

Cahn, J. H., Kaler, J. B., \& Stanghellini, L. 1992, A\&AS, 94, 399

Condon, J. J., Cotton, W. D., Greisen, E. W., et al. 1998, AJ, 115,1693

Corradi, R. L. M., \& Schwarz, H. E. 1995, A\&A, 293, 871

Esteban, C., Peimbert, M., Torres-Peimbert, S., \& Escalante, V. 1998, MNRAS, 295, 401 
Fluks, M. A., Plez, B., de Winter, D., et al. 1994, A\&AS, 105, 311

Grasdalen, G. L. 1979, ApJ, 229, 587

Grevesse, N., \& Noels, A. 1993, in Origin of the Elements, ed. N. Prantos, et al. (Camb. Univ. Press), 15

Hajian, A. R., \& Terzian, Y. 1996, PASP, 108, 419

Harrington, J. P., Seaton, M. J., Adams, S., \& Lutz, J. H. 1982, MNRAS, 199, 517

Hummer, D. G., \& Storey, P. J. 1987, MNRAS, 224, 801

Hyung, S., \& Aller, L. H. 1997a, ApJ, 491, 242

Hyung, S., \& Aller, L. H. 1997b, MNRAS, 292, 71

Isaacman, R. 1984, MNRAS, 208, 399

Kingsburgh, R. L., \& Barlow, M. J. 1994, MNRAS, 271, 257

Liu, X.-W., Barlow, M. J., Cohen, M., et al. 2001, MNRAS, 323,343
Phillips, J. P., \& Mampaso, A. 1988, A\&A, 190, 273

Pottasch, S. R., \& Beintema, D. A. 1999, A\&A, 347, 974

Pottasch, S. R., Beintema, D. A., \& Feibelman, W. A. 2000, A\&A, 363, 767

Pottasch, S. R., Preite-Martinez, A., Olnon, F. M., et al. 1986, A\&A, 161, 363

Rowlands, N., Houck, J. R., Skrutskie, M. F., \& Shure, M. 1993, PASP, 105, 1287

Shure, M. A., Herter, T., Houck, J. R., et al. 1983, ApJ, 270, 645

Stasinska, G., Tylenda, R., Acker, A., \& Stenholm, B. 1992, A\&A, 266, 486

Zijlstra, A., Pottasch, S. R., \& Bignell, C. 1989, A\&AS, 79, 329 